\title{
Dynamic Locomotion Gaits of a Compliantly Actuated Quadruped with SLIP-like Articulated Legs Embodied in the Mechanical Design
}

\author{
Dominic Lakatos ${ }^{1}$, Kai Ploeger ${ }^{1}$, Florian Loeff ${ }^{1}$, Daniel Seidel ${ }^{1,2}$, Florian Schmidt ${ }^{1}$, Thomas Gumpert ${ }^{1}$, \\ Freia John ${ }^{3}$, Torsten Bertram ${ }^{3}$, and Alin Albu-Schäffer ${ }^{1,2}$
}

\begin{abstract}
The spring loaded inverted pendulum (SLIP) model has been extensively shown to be fundamental for legged locomotion. However, the way this low-order template model dynamics is anchored in high-dimensional articulated multibody systems describing compliantly actuated robots (and animals) is not obvious and has not been shown so far. In this paper, an articulated leg mechanism and a corresponding quadrupedal robot design are introduced, for which the natural oscillation dynamics is structurally equivalent to the SLIP. On the basis of this property, computationally simple and robust control methods are proposed, which implement the gaits of pronking, trotting, and dynamic walking in the real robotic system. Experiments with a compliantly actuated quadruped featuring only low performance electrical drives validate the effectiveness of the proposed approach.
\end{abstract}

Index Terms-Compliance and Impedance Control, Legged Robots, Dynamics Embodiment, Spring Loaded Inverted Pendulum

\section{INTRODUCTION}

$\mathbf{T}$ HE benefits of springs in legged locomotion have been validated in the conceptual work of Alexander [1]. The hypothesis that the high-dimensional, nonlinear dynamics of complex legged animals collapses to template models of strongly reduced order, like the spring loaded inverted pendulum (SLIP) model [2] and extensions [3], [4], [5], [6], [7], is further supported by experimental results [8]. In particular, the authors of the review article [9] hypothesize that embodying these template models as invariant (and attracting) submanifolds into the high order multibody dynamics of articulated legged systems is crucial to energetically efficient and performant locomotion.

The fastest mammals on earth are quadrupeds. ${ }^{1}$ As discussed above, it is very likely that such a high performance

Manuscript received: February, 24, 2018; Revised May, 30, 2018; AcceptedJune, 27, 2018.

This paper was recommended for publication by Editor Paolo Rocco upon evaluation of the Associate Editor and Reviewers' comments. This work was supported by (organizations/grants which supported the work.)

${ }^{1}$ D. Lakatos, K. Ploeger, F. Loeffl, D. Seidel, F. Schmidt, T. Gumpert, and A. Albu-Schäffer are with the Robotic Mechatronic Center (RMC), Institute of Robotics and Mechatronics, German Aerospace Center (DLR), D-82234 Oberpfaffenhofen, Germany dominic.lakatosedlr.de

${ }^{2}$ D. Seidel and A. Albu-Schäffer are also with Technical University Munich, Chair of Sensor Based Robots and Intelligent Assistance Systems, Department of Informatics, D-85748 Garching, Germany

${ }^{3}$ F. John and T. Bertram are with the Institute of Control Theory and Systems Engineering, TU Dortmund University, D-44227 Dortmund, Germany

Digital Object Identifier (DOI): see top of this page.

${ }^{1}$ The cheetah can reach a peak velocity of $120 \mathrm{~km} / \mathrm{h}$ and the antelope approaches a maximum speed of $88 \mathrm{~km} / \mathrm{h}$ over a distance of $800 \mathrm{~m}$.

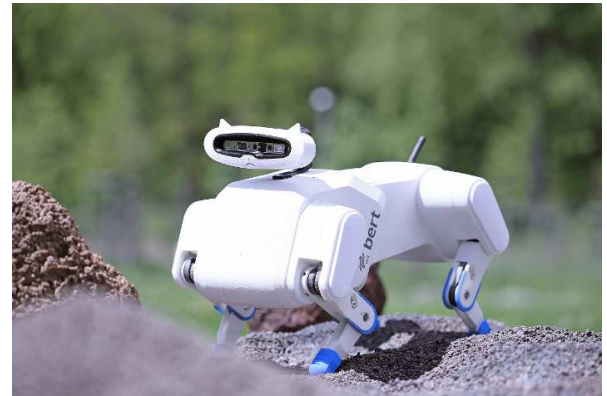

Fig. 1. Compliantly actuated quadruped with SLIP-like articulated legs.

is the result of a very optimized, system inherent locomotion dynamics. In the last decades, numerous quadrupedal robots have been developed, which are based upon hydraulic/pneumatic actuators [10], [11], [12], [13], electrically powered direct drives [14], and compliantly actuated systems [15], [16], [17]. ${ }^{2}$ The quadruped reported in [10] is composed of hydraulically/pneumatically powered telescopic legs, which closely resemble the dynamics of a spring-mass system. On the basis of such a system, [18], [19] found fundamental control principles of legged locomotion and demonstrated to perform effectively in experiments. These findings are further augmented and successfully validated on systems, which exploit also the advantageous properties of segmented legs such as versatility regarding locomotion in uneven terrain [11], [20], [21], [22]. All the mentioned quadrupedal robots have demonstrated remarkable dynamic walking and running performance. Thereby, a common approach is to implement a certain interaction or virtual model behavior by joint torque control, while exploiting physical elasticities mainly to absorb high frequency external forces, which occur due to ground impacts of the feet. An exception is MIT's cheetah [14] with its electrical direct drives of low inertia and friction. As a result of electric energy storage capabilities and an very optimized power train, MIT's cheetah achieves already high energetic efficiency. However, all above mentioned robotic applications of versatile and dynamic legged locomotion require high bandwidth joint torque interfaces, which are technically costly, expensive and rather implausible from a biological point of view $^{3}$.

\footnotetext{
${ }^{2}$ This is only a representative selection of quadrupedal robots.

${ }^{3}$ Transmission delays in biological motor control systems complicate high bandwidth torque control.
} 


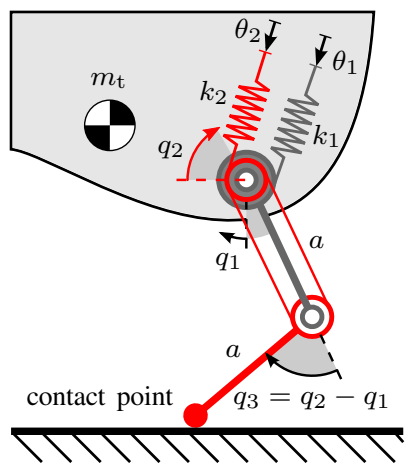

(a)

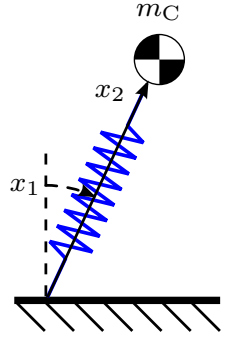

(b)
Fig. 2. Matching the dynamics of a two-segment leg to the SLIP model: (a) two-segment leg; (b) SLIP.

In our previous work [23], the theory of so-called eigenmodes of nonlinear dynamics and a corresponding embodiment procedure are proposed. This paper presents a compliantly actuated quadrupedal robot with articulated legs featuring a SLIP-like dynamical behavior embodied as eigenmodes in the mechanical system design. For configurations in which the system displays full symmetry, any robot will display this behaviour. This is validated for the quadrupedal robot at hand. However, the implemented (vertebrates-like) segmented leg design features this behavior even for a single leg in contact, i. e., in a non-symetric configuration. (The implemented leg design features a SLIP-like dynamics based upon the assumption that the leg mass is negligible compared to the trunk, i. e., the mass of one leg is lower than $5 \%$ of the total mass.) This is particularly advantageous for dynamic gaits, where in some phases of the gait cycle only one leg is in stance. On the basis of these dynamics properties, simple and robust control methods are proposed, which implement the gaits of pronking, trotting, and dynamic walking although the performance of the electrical drives, as used in the robot, is low.

\section{ARTICULATED LEG DESIGN WITH SLIP-LIKE DYNAMICS EMBODIED}

At first view, the dynamics of an articulated mechanism, as e. g., shown in Fig. 2(a) and of the SLIP-like model (Fig. 2(b)) is expected to differ substantially. However, using the method of modal dynamics matching [23], kinematic, inertial and elastic parameters can be found such that they match. This is demonstrated for a mechanically straightforwardly implementable example.

Consider the structural model of a two-segment leg during stance (Fig. 2(a)). The leg is assumed to be attached to the main body (trunk) with very high inertial properties such that its rotation can be neglected, i. e., the trunk has only the two translational degrees of freedom of the sagittal plane. ${ }^{4}$ The thigh is connected to the trunk by a rotational joint with coordinate $q_{1}$. The shank is hinged to the thigh with relative

\footnotetext{
${ }^{4}$ Note that this assumption holds especially for quadrupeds, where the fore and hind legs are configured symmetrically and the center of mass (COM) of the trunk is located at the center of pivot points of the legs.
}

coordinate $q_{3}$. There is a pulley concentric with the hip joint with relative coordinate $q_{2}$, which couples to the knee joint such that $q_{3}=q_{2}-q_{1}$. A point-foot is considered, which is constrained during stance phases to touch the ground such that the configuration of the system is determined by the minimum set of configuration coordinates $\boldsymbol{q}=\left(q_{1}, q_{2}\right) \in \mathbb{R}^{2}$. Assuming that each leg segment has equal length $a>0$ and equal mass $m_{1}>0$ concentrated at the segment center, and assuming further that hip joint and pulley are actuated via linear springs with spring constants $k_{1}>0$ and $k_{2}>0$, and actuator positions $\theta_{1}$ and $\theta_{2}$, the dynamics of the structural two-segment leg model can be expressed in the form

$$
\boldsymbol{M}(\boldsymbol{q}) \ddot{\boldsymbol{q}}+\boldsymbol{C}(\boldsymbol{q}, \dot{\boldsymbol{q}}) \dot{\boldsymbol{q}}=-{\frac{\partial U_{\mathrm{g}}(\boldsymbol{q})^{T}}{\partial \boldsymbol{q}}}^{T}-\boldsymbol{K}(\boldsymbol{q}-\boldsymbol{\theta})
$$

where the $2 \times 2$ inertia and stiffness matrices have the form

$$
\begin{array}{rlr}
\boldsymbol{M}(\boldsymbol{q}) & =a^{2}\left[\begin{array}{cc}
m_{\mathrm{t}}+\frac{m_{1}}{4} & \left(m_{\mathrm{t}}+\frac{m_{1}}{2}\right) \cos \left(q_{2}-q_{1}\right) \\
\operatorname{sym} . & m_{\mathrm{t}}+\frac{5 m_{1}}{4}
\end{array}\right], \\
\boldsymbol{K} & =\left[\begin{array}{cc}
k_{1} & 0 \\
0 & k_{2}
\end{array}\right] .
\end{array}
$$

Moreover, $\quad \boldsymbol{C}(\boldsymbol{q}, \dot{\boldsymbol{q}}) \dot{\boldsymbol{q}}$ denotes the generalized Coriolis/centrifugal force and $U_{\mathrm{g}}(\boldsymbol{q})$ represents the gravitational potential.

The goal is to match the dynamics, as described above, to the one of the SLIP model. Consider therefore the stance phase dynamics of the SLIP model expressed in polar coordinates,

$$
\begin{gathered}
m_{\mathrm{C}}\left\{\left[\begin{array}{cc}
x_{2}^{2} & 0 \\
0 & 1
\end{array}\right]\left(\begin{array}{l}
\ddot{x}_{1} \\
\ddot{x}_{2}
\end{array}\right)+x_{2}\left[\begin{array}{cc}
\dot{x}_{2} & \dot{x}_{1} \\
-\dot{x}_{1} & 0
\end{array}\right]\left(\begin{array}{l}
\dot{x}_{1} \\
\dot{x}_{2}
\end{array}\right)+\right. \\
\left.g_{0}\left(\begin{array}{c}
-\sin \left(x_{1}\right) x_{2} \\
\cos \left(x_{1}\right)
\end{array}\right)\right\}=-\left(\begin{array}{c}
0 \\
\frac{\partial U_{\mathrm{e}}\left(x_{2}-r_{0}\right)}{\partial x_{2}}
\end{array}\right) .
\end{gathered}
$$

As schematically sketched in Fig. 2(b), $\boldsymbol{x} \in \mathbb{R} \times \mathbb{R}_{\geq 0}$ denotes the position of the mass $m_{\mathrm{C}}$ w.r.t. to the pivot point on the ground expressed in a polar coordinate system. Thereby, $x_{1}$ represents the polar angle and $x_{2}$ denotes the radius. Due to this choice of coordinates, the elastic potential $U_{\mathrm{e}}\left(x_{2}-r_{0}\right)$ depends only on the displacement in the radial direction (w. r. t. to the rest length $r_{0}>0$ ). Note that the nonlinear SLIP dynamics features an eigenmode $\mathcal{W}:=\left\{\boldsymbol{x} \in \mathbb{R} \times \mathbb{R}_{\geq 0} \mid x_{1}=0\right\}$ according to Definition 1 (see, Appendix).

To match the structural dynamics model of the two-segment leg (1)-(3) to the desired SLIP dynamics (4), (1)-(3) is transformed under the change of coordinates

$$
\hat{\boldsymbol{x}}=\boldsymbol{f}(\boldsymbol{q})=\left(\frac{\frac{q_{1}+q_{2}}{2}}{a \sqrt{2\left(1+\cos \left(q_{2}-q_{1}\right)\right)}}\right) .
$$

Thereby, $\hat{\boldsymbol{x}} \in \mathbb{R} \times[0 ; 2 a]$ denotes the position of the hip w. r. t. to the contact point in polar coordinates. Then, choosing the mass of the legs $m_{1}$ and the ratio of stiffness $k_{2} / k_{1}$ as design parameters, i.e., $\boldsymbol{\zeta}=\left(m_{1}, k_{2} / k_{1}\right)$, it is found that global matching of eigenvectors is achieved if $m_{1}=0$ and $k:=k_{1}=k_{2}$, i.e., $\hat{\boldsymbol{\zeta}}=(0,1)$. Substituting these design 
parameters in (1)-(3) and transforming the resulting dynamics under the change of coordinates $(5)$, yields

$$
\begin{aligned}
& m_{\mathrm{t}}\left\{\left[\begin{array}{cc}
\hat{x}_{2}^{2} & 0 \\
0 & 1
\end{array}\right]\left(\begin{array}{l}
\ddot{\hat{x}}_{1} \\
\ddot{\hat{x}}_{2}
\end{array}\right)+\hat{x}_{2}\left[\begin{array}{cc}
\dot{\hat{x}}_{2} & \dot{\hat{x}}_{1} \\
-\dot{\hat{x}}_{1} & 0
\end{array}\right]\left(\begin{array}{l}
\dot{\hat{x}}_{1} \\
\dot{\hat{x}}_{2}
\end{array}\right)+\right.
\end{aligned}
$$

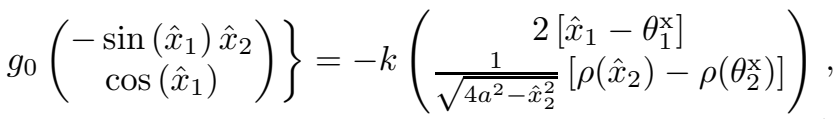

where $\rho(y):=\mp \arccos \left(1-\frac{y^{2}}{2 a^{2}}\right)$ represents an abbreviation related to the knee angle $q_{3}=q_{2}-q_{1}=$ $\mp\left[\arccos \left(1-\frac{\hat{x}_{2}^{2}}{2 a^{2}}\right)-\pi\right]$. Thereby, the negative or positive sign selects the solutions $q_{3}>0$ or $q_{3}<0$, respectively, and $\boldsymbol{\theta}^{\mathrm{x}}=\boldsymbol{f}(\boldsymbol{\theta})$ denotes the transformed control input. Indeed, by equating $m_{\mathrm{t}}=m_{\mathrm{C}}$ and $\hat{\boldsymbol{x}}=\boldsymbol{x}$, it can be seen that the inertial dynamics of the matched two-segment leg model (6) and the slip model (4) are equivalent. This is not very surprising, since the inertia of the leg segments is set to zero, i.e., $m_{1}=0$. Note that the example of [23] does not require to make this assumption, but the mechanism is significantly more complex. A prototype of such a pantograph leg is currently being designed. The assumption of zero leg mass, as made in (6), is consistent with the common wisdom of designing the leg segments as light-weight as possible (cf. the leg design in [24], which places the actuators also in the trunk and implements a spring acting in the direction of the leg axis), and results in a much less complex mechanical design (compared to the example of [23]). In particular, the decoupled structure of the elasticity as in the SLIP model (4) is maintained for the segmented leg, cf. (6). For $\theta_{1}^{\mathrm{x}}=0$, the nonlinear dynamics of the two-segment leg (6) features an eigenmode $\hat{\mathcal{W}}:=\left\{\hat{\boldsymbol{x}} \in \mathbb{R} \times[0 ; 2 a] \mid \hat{x}_{1}=0\right\}$ for any $\theta_{2}^{\mathrm{x}} \in[0 ; 2 a]$. Note that for a symmetric positioning of legs and a symmetric distribution of the trunk inertia, the eigenmode $\hat{\mathcal{W}}$ can be maintained for the complete quadruped, even in case of two-segment legs with non-zero inertias. This is validated after introducing the quadrupedal system design.

\section{QUADRUPEDAL SYSTEM DESIGN}

The proposed quadrupedal design aims at featuring dynamic locomotion capabilities based upon low performance (and low-cost) servo drives. For the dimensioning, a single leg is considered. Thereby, the task of vertical jumping serves as a reference. The off-the-shelf high torque servo Savöx $\mathrm{SV}-1270 \mathrm{TG}$ is selected as servo motor for the series elastic actuators (SEA). In order to increase the control performance of the drive and to allow to connect an additional sensor (to measure the spring deflection and the output position of the SEA), the build-in electronics of the off-the-shelf servo is replaced by a customized one. The resulting servo drive features a maximum (active) torque of $1.3 \mathrm{Nm}$ and a maximum angular velocity of $10.0 \mathrm{rad} / \mathrm{s}$. The selection of the servo motor yields an estimate of the main body weight. Given the maximum torque, velocity, and an estimate of the weight of the single-leg system, the leg-segment lengths and spring stiffnesses are determined in a series of computer simulation based optimizations of the jumping height. In this procedure,

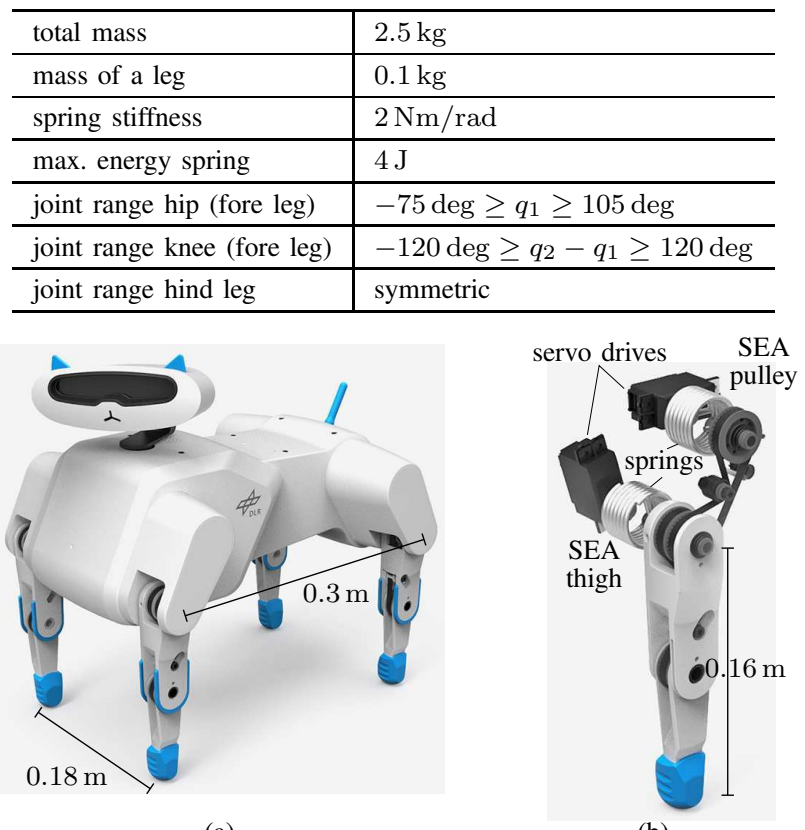

(a)

(b)

Fig. 3. Compliantly actuated quadrupedal robot Bert: (a) dimensions; (b) leg mechanism.

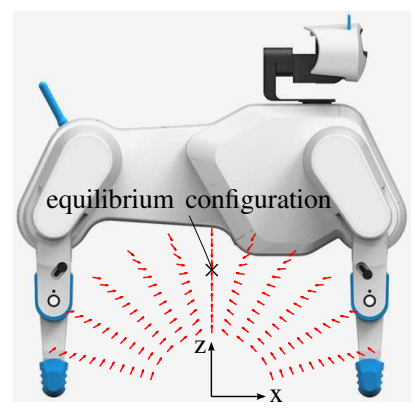

(a)

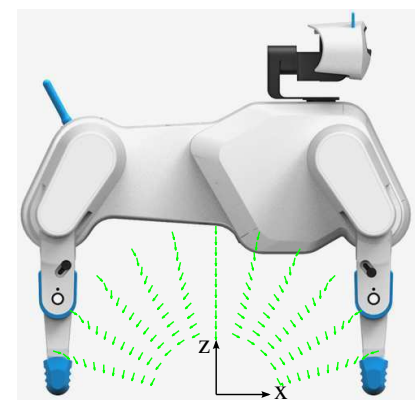

(b)
Fig. 4. Acceleration field of the compliantly actuated quadruped Bert in stance: (a) due to potential forces: $\ddot{\boldsymbol{q}}_{\mathrm{pot}}=-\boldsymbol{M}(\boldsymbol{q})^{-1} \partial U(\boldsymbol{q}) / \partial \boldsymbol{q}$; (b) Coriolis/centrifugal accelerations: $\ddot{\boldsymbol{q}}_{\mathrm{CC}}=-\boldsymbol{M}(\boldsymbol{q})^{-1} \boldsymbol{C}(\boldsymbol{q}, \dot{\boldsymbol{q}}) \dot{\boldsymbol{q}}$. It can be observed that the acceleration due to potential forces as well as the Coriolis/centrifugal acceleration are aligned with the $\mathrm{z}$-axis for a motion (displacement and velocity) along the z-axis. Thus, the z-axis represents an invariant set according to Definition 1 in the Appendix.

the insights of the modal leg design, as derived above, are taken into account such that only two parameters (i.e., the length of thigh and shank $a$ and the stiffness of both actuated degrees of freedom $k$ ) need to be found. In order to maximally exploit the potential energy storing capabilities of SEAs, it is assumed that very low, viscous damping acts only at link side. This assumption reveals the requirement to implement the SEA with as few friction as possible in parallel to the spring. In order to also satisfy the low weight assumption made for the simulation model, the SEA is realized by a torsional spring. The resulting mechanical implementation of the compliantly actuated, modal leg design, as shown in Fig. 3(b), is also advantageous regarding the installation space, and determines the width of shoulder and hip of the quadruped (Fig. 3(a)). The distance of fore hip and hind hip is selected such that the 
gallop gait is possible, i. e., fore-feet and hind-feet motions can intersect.

The arrangement of the legs as well as the inertial properties of the main body are chosen to be symmetric such that the complete quadrupedal system features an eigenmode, which can be exploited for vertical bouncing motions. This can be validated by investigating the acceleration fields for displacements w.r.t. to an equilibrium configuration of the eigenmode and the field of Coriolis/centrifugal accelerations as shown in Fig. 4(a) and 4(b), respectively. In more detail, the task dynamics of the complete quadrupedal system is considered in a stance phase configuration, where all four feet are constrained at positions on the ground perpendicular to hip and shoulder rotation axes ${ }^{5}$. A symmetric equilibrium configuration is selected, in which the main body is parallel to the ground plane. The condition of Definition 1 related to potential forces is tested by analyzing the direction of acceleration for translational displacements of the trunk w. r.t. a ground fixed coordinate system (Fig. 4(a)). The condition of Definition 1 related to Coriolis/centrifugal forces needs to be satisfied simultaneously. That is, when the system moves in a certain direction (i.e., the velocity magnitude is non-zero), an acceleration results due to the Coriolis/centrifugal forces. In order that the system continues moving along the same linear direction, the accelerations due to Coriolis/centrifugal and potential forces need to be aligned with the velocity as well as the displacement. This is tested by evaluating the direction of the Coriolis/centrifugal acceleration for different velocities expressed in polar coordinates at several configurations (Fig. 4(b)). Thereby, the directions of the velocities correspond to the relative displacements of the robot w.r.t. the initial configuration considered for the potential force test. For instance, if the relative displacement is solely polar (angular), then also the tested velocity consists only of an angular component. Both fields display vertical lines of accelerations such that the existence of an eigenmode can be deduced according to Definition 1 (Appendix).

\section{CONTROL OF DYNAMiC LOCOMOTION GAITS}

The dynamics of multi-legged locomotion such as pronking and trotting can be approximated by a spring-mass model [25]. As validated above (cf. Fig. 4(a) and 4(b)), for symmetric leg configurations and contact situations, the compliant quadruped at hand features an oscillation mode, which corresponds to such bouncing motions. This intrinsic dynamics behavior can be directly exploited in the stance phase and combined with well-studied foot-placement strategies [18] to achieve dynamic locomotion gaits. However, the spring-mass or SLIP model is conservative, while the dynamics of the real quadrupedal system is subject to energetic losses due to impacts and friction in the joints. To excite and sustain the intrinsic oscillatory behavior of the real plant, a control method based on switching of motor positions can be applied along the eigenmodes. Accordingly, the proposed gait controllers switch the position

\footnotetext{
${ }^{5}$ Since all contact points of the legs can move only in parallel planes, only 10 rather than 12 constraints are feasible, i. e., all four contact points are constrained in vertical and forward/backward direction, but only either the two left or right feet are constrained in lateral direction.
}

of the motors triggered by events which depend solely on states at position level.

\section{A. Pronking}

A fundamental difference of the SLIP and the modal leg dynamics is that for the latter, the polar angle $\hat{x}_{1}$ is statically controllable during stance (cf. the right hand sides of (4) and (6)). This additional input variable $\theta_{1}^{\mathrm{x}}$ can be utilized to control the pendulum motion of the trunk in a pronking gait. In [26], it has been demonstrated for a single-leg system that the simple switching law

$$
\left\{\begin{array}{lll}
\boldsymbol{\theta}\left(\boldsymbol{\theta}_{-}, \boldsymbol{q}\right)= \\
\boldsymbol{f}^{-1}\left(-\alpha_{1}, r_{0}\right)+\boldsymbol{w} \hat{s} & \text { if } & \boldsymbol{w}^{T}{\frac{\partial U_{\mathrm{e}}\left(\boldsymbol{\theta}_{-}, \boldsymbol{q}\right)}{\partial \boldsymbol{q}}}^{T}>\epsilon_{\tau_{\mathrm{w}}} \\
\boldsymbol{f}^{-1}\left(\alpha_{1}, r_{0}\right) & \text { if } & \boldsymbol{w}^{T} \frac{\partial U_{\mathrm{e}}\left(\boldsymbol{\theta}_{-}, \boldsymbol{q}\right)}{\partial \boldsymbol{q}}{ }^{T}<\epsilon_{\tau_{\mathrm{w}}}
\end{array},\right.
$$

(where $\boldsymbol{f}^{-1}$ denotes the inverse mapping of (5) and $\boldsymbol{\theta}_{-}$is the state of $\boldsymbol{\theta}$ before the switching instance) suffices to stabilize a periodic hopping motion. The control (7) switches only between two equilibrium configurations. The corresponding motor positions are parametrized in polar coordinates by the landing angle $\alpha_{1} \in(-\pi / 2 ; \pi / 2)$, the radial rest length $r_{0}>0$, and the switching amplitude $\hat{s}>0$ along the eigenvector

$$
\boldsymbol{w}=\operatorname{sign}\left(q_{2}-q_{1}\right)\left(\begin{array}{ll}
1 & -1
\end{array}\right)^{T} .
$$

The transitions between the two controller states are triggered by approximating the touchdown and takeoff event by thresholding the generalized elastic force on the eigenmode $\boldsymbol{w}^{T}{\frac{\partial U_{\mathrm{e}}\left(\boldsymbol{\theta}_{-}, \boldsymbol{q}\right)}{\partial \boldsymbol{q}}}^{T}$ w.r.t. a constant $\epsilon_{\tau_{\mathrm{w}}}>0$ from below (touchdown) and above (takeoff), respectively. Therefore, the complete control is determined by only four, intuitive parameters. In particular, the parameter corresponding to the angle of attack $\alpha_{1}$ can be considered to regulate the average locomotion velocity $v$ w.r.t. a desired value $v_{\text {des }}$, e. g., by an iterative law of the form

$$
\alpha_{1}(j)=\alpha_{1}(j-1)+k_{\mathrm{v}}\left(v(j)-v_{\mathrm{des}}\right),
$$

which updates $\alpha_{1}$ once per jumping cycle $j$ with a low gain $k_{\mathrm{v}}>0$. The control (9) is similar to the step-length adaptation as proposed by Raibert [10]. The difference w. r. t. Raibert's controller is that (9) cumulates the control-error instead of considering a proportional and feed-forward term, as proposed in [18].

The single-leg hopping control (7) can be directly transfered to the quadrupedal system by "linking" the input of all legs to a single, "virtual" leg, as proposed in [18]. In case of pronking on spot, this results in a motion, which evolves ideally in the eigenmode of the plant, although the quadrupedal system is composed of articulated legs with non-zero mass. ${ }^{6}$ As such, this pronking control is potentially efficient w. r. t. the jumping height. However, if the goal is to travel forward, there are different quadrupedal gaits, which are way more efficient depending on the desired speed [27].

\footnotetext{
${ }^{6}$ This is based on the assumption that the quadrupedal system is symmetric w. r. t. the vertical axis.
} 


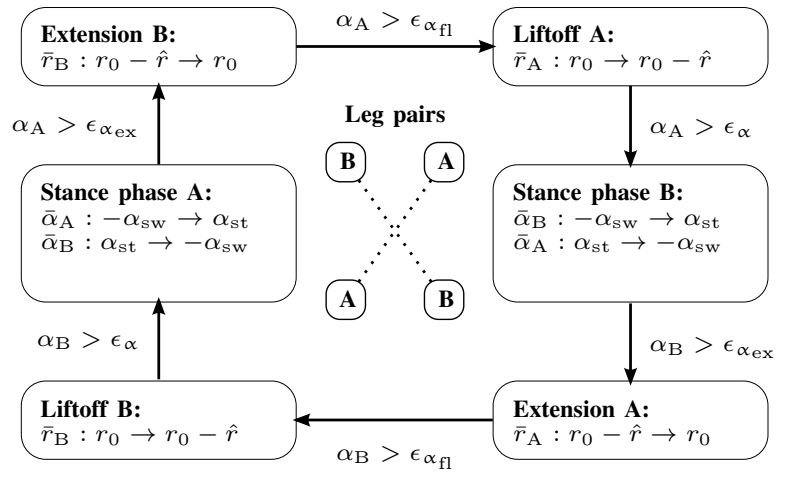

Fig. 5. Finite state machine controlling quadrupedal trot. Equilibrium configurations (motor positions) and link configurations in polar coordinates are denoted $\left(\bar{\alpha}_{i}, \bar{r}_{i}\right)=\boldsymbol{f}\left(\boldsymbol{\theta}_{i}\right)$ and $\left(\alpha_{i}, r_{i}\right)=\boldsymbol{f}\left(\boldsymbol{q}_{i}\right)$ according to (5) respectively, where the index $i=\{\mathrm{A}, \mathrm{B}\}$ refers to diagonally "linked" leg pairs. The transition to the liftoff state of leg pair $\mathrm{A}$ is triggered when the link-side leg angle $\alpha_{\mathrm{A}}$ is greater than the threshold value $\epsilon_{\alpha_{\mathrm{fl}}}$. In this state, the reference rest length $r_{0}$ is reduced by an amount of $\hat{r}$. When the leg angle $\alpha_{\mathrm{A}}$ further hits the threshold value $\epsilon_{\alpha}$ from below, the reference angle of leg pair A is changed to $-\alpha_{\mathrm{Sw}}$ and the reference angle of leg pair B is changed to $\alpha_{\text {st }}$ such that $\mathrm{A}$ and $\mathrm{B}$ swing forward and backward, respectively. The extension of the new leg pair in stance B (to the reference rest length $r_{0}$ ) is triggered when the link-side leg angle of the indicated pair $\alpha_{\mathrm{B}}$ is greater than $\epsilon_{\alpha_{\mathrm{ex}}}$.

\section{B. Trotting}

The trot control, which will be proposed in the following, serves as an example to show how the natural oscillatory dynamics of the quadrupedal system can be exploited to achieve forward locomotion. The running trot is a dynamic gait, where diagonal pairs of legs move in phase. While one leg pair is in stance and transports the center of mass (COM) forward in locomotion direction, the other leg pair swings towards the touchdown configuration. The gait can display a flight phase in between the alternation of these functionalities. In that case, the total COM needs to oscillate in vertical direction, since during the flight phase, the height of the total COM cannot be constant due to gravity. However, from a view point of energetic efficiency (regarding forward locomotion), the vertical oscillation amplitude needs to be kept as low as possible, since due to friction (which is present in any physical system), any radial motion of the stance legs is subject to energetic losses, although kinetic energy (of the flight phase) is partially stored in the corresponding springs. This observation reveals that a potential source to improve efficiency is given in the generation of the polar leg motion, which motivates a controller design based upon the natural oscillatory dynamics predominantly in this polar direction of the legs, cf. (6). ${ }^{7}$

The gait control can be described by a finite state machine (FSM), which switches the equilibrium configurations of the legs (Fig. 5). The link and motor positions of all legs are expressed in polar coordinates (5). Diagonal leg pairs are considered as "linked". The gait generation is based on the idea to excite an antiphasic, elastic pendulum motion of the stance and swing leg pair by moving the motors as few as possible. This can be achieved by switching the polar

${ }^{7}$ Note that for implementation based on the SLIP dynamics, e. g., via virtual model control [28], the motors performing the polar leg motion would require to move as fast as the corresponding links, since there is no elasticity directly acting in the polar direction to perform the movement.

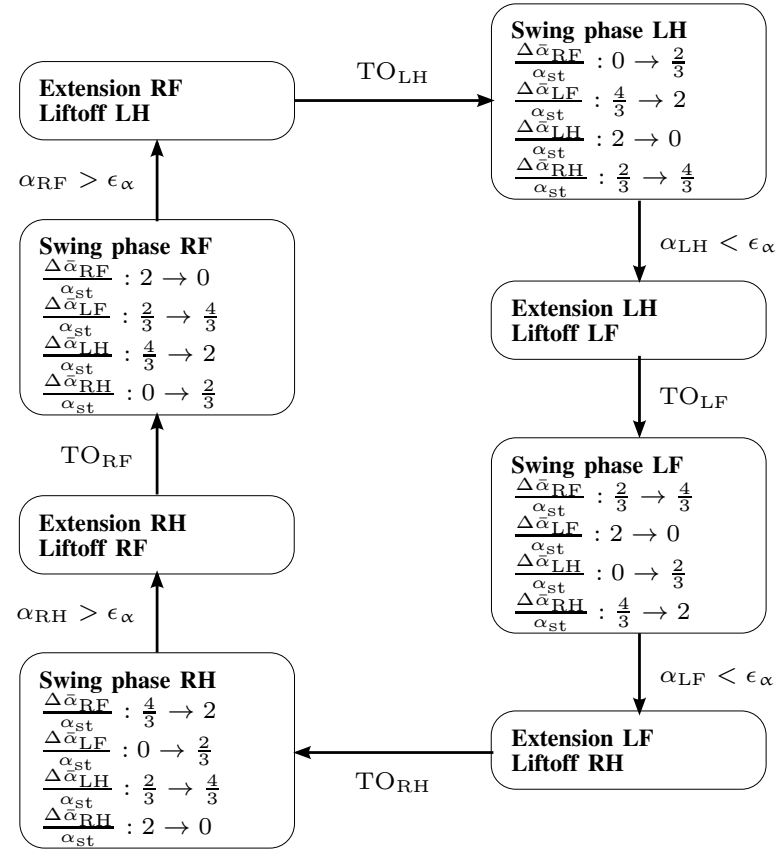

Fig. 6. Finite state machine controlling quadrupedal dynamic walking. Equilibrium configurations (motor positions) and link configurations in polar coordinates are denoted $\left(\bar{\alpha}_{i}, \bar{r}_{i}\right)=\boldsymbol{f}\left(\boldsymbol{\theta}_{i}\right)$ and $\left(\alpha_{i}, r_{i}\right)=\boldsymbol{f}\left(\boldsymbol{q}_{i}\right)$ according to (5), respectively, and the takeoff event is denoted by $\mathrm{TO}_{i}$, where the index $i=\{\mathrm{RF}, \mathrm{LF}, \mathrm{LH}, \mathrm{RH}\}$ refers to the legs. Note that the commanded equilibrium configuration is $\bar{\alpha}_{i}=\alpha_{\mathrm{a}}+\Delta \bar{\alpha}_{i}$. Therefore, e. g., the takeoff event of the left hind leg $\mathrm{TO}_{\mathrm{LH}}$ triggers its swing phase, i. e., the reference angle $\bar{\alpha}_{\mathrm{LH}}$ is changed from $\alpha_{\mathrm{a}}+2 \alpha_{\mathrm{st}}$ (foot most backward) to $\alpha_{\mathrm{a}}$ (foot most forward). The reference angles of the remaining legs are shifted by one third of the angular step length $2 \alpha_{\text {st }}$ such that the feet move backward. The extension of the left hind leg and the liftoff of the left fore leg is initiated, when the link-side angle of the swing leg $\alpha_{\mathrm{LH}}$ is lower than the threshold value $\epsilon_{\alpha}$ (i. e., when the swing foot has reached its desired forward position).

equilibrium configuration of the stance and swing legs to $\alpha_{\mathrm{st}}$ and $-\alpha_{\mathrm{sw}}$, respectively. Thereby, the sum of the parameters $\alpha_{\mathrm{st}} \geq 0$ and $\alpha_{\mathrm{sw}} \geq 0$ determines the static polar step length. The current stance phase is initiated depending on the polar angle of the previous leg pair in stance. Thereby, the threshold $\epsilon_{\alpha}>0$ determines also the actual dynamic step length. It becomes evident that there might be an optimal value for $\epsilon_{\alpha}$, which for a given static step length (which determines the switching amplitude) maximizes the actual dynamic step length. In between the main two (finite) states of the stance phase, two more states are required, which initiate the radial extension and flexion of the swing and stance leg, respectively. Since the effective inertia of the stance legs is much larger than that of the swing legs but the stiffness is equal, also the time constant of oscillation of the stance legs is larger. Loosely speaking, this means that the swing legs move faster than the ones in stance. As such, the extension of the swing legs needs to be initiated before the legs in stance are lifted. This can be achieved by setting the rest length of the swing legs to the initial leg length $r_{0} \in\left(0 ; r_{\max }\right]$, when the polar angle of the legs in stance hits the threshold $\epsilon_{\alpha_{\mathrm{ex}}}<\epsilon_{\alpha}$ from below. The liftoff is initiated by reducing the initial rest length of the stance legs by an amount of $\hat{r} \in\left(0 ; r_{0}\right)$, when the polar angle of the same leg pair crosses the threshold $\epsilon_{\alpha_{\mathrm{fl}}}>\epsilon_{\alpha_{\mathrm{ex}}}$ (where $\epsilon_{\alpha_{\mathrm{fl}}}<\epsilon_{\alpha}$ ) from below. Note that for the legs in 
stance, their rest length is only decreased, which means that at most the potential energy is removed in the radial direction. However, although the radial oscillation is not directly excited, the energy storing capabilities in vertical direction can be exploited by selecting $\alpha_{\mathrm{st}}>\alpha_{\mathrm{sw}}$.

\section{Dynamic walking}

Quadrupedal walking is a gait for rather moderate locomotion speeds with increased stability against falling (compared to pronking or trotting). The compliantly actuated quadrupedal robot, as introduced in Sect. III, is designed for highly dynamical locomotion gaits. In the following, a dynamic walking control is proposed, which aims at validating that the same robotic system is also able to perform such "less dynamical" but more stable (regarding falling) gaits. Quadrupedal walking is a cyclic motion, where each of the four legs touches down and lifts off subsequently. Consequently, the legs move shifted by one fourth of the step length, and the feet touch down in the order: left hind (LH), left fore (LF), right hind ( $\mathrm{RH})$, and right fore (RF). To achieve a dynamic walking, each foot is lifted right before the previous swing leg touches the ground. This leads to phases, where only two legs are in stance. Therefore, the gait pattern is still not always statically stable.

Due to the SLIP-like dynamics properties of the legs, as proposed in Sect. II, the dynamic walking control can be implemented by a FSM, which switches the equilibrium configurations of the legs in polar coordinates triggered by state-dependent events (Fig. 6). For instance, starting in the swing phase of the RF leg, the extension of the RF to the nominal rest length $r_{0}>0$ and the liftoff of the LH (i.e., decrease in rest length by $\left.\hat{r} \in\left(0 ; r_{0}\right)\right)$ is initiated, as soon as the polar angle of the swing leg (RF) crosses the threshold $\epsilon_{\alpha}$ from below. The swing phase of the LH leg is triggered by its takeoff event, etc. Thereby, the locomotion speed is determined by the nominal polar step length $2 \alpha_{\text {st }}>0$ and the angle of attack $\alpha_{\mathrm{a}}$. Note that therefore the complete walking control is parametrized by only five parameters.

\section{EXPERIMENTS}

The performance of the quadrupedal pronk, trot, and dynamic walking controllers, as proposed in Sect. IV, are validated in real hardware experiments with the compliantly actuated quadruped introduced in Sect. III. In more detail, a PD-controller for the position of the servo drives is implemented via pulse width modulation (PWM) on the servo units, which communicate with a central single board computer (Odroid XU4), i.e., the servo units receive motor position commands and send measured motor and link positions at a rate of $1 \mathrm{kHz}$. Additionally, the position and orientation of the main body is measured by an optical tracking system (not used in the control loop). In the current version, the quadrupedal system is powered externally via a cable, while high-level communication such as starting/stopping FSM and data logging is realized via a wireless Ethernet connection.

\section{A. Pronking}

The pronk gait can be used to overcome obstacles. A reasonable performance measure takes also the jumping height into account. In the experimental evaluation of the pronking controller, the jumping distance is considered as performance measure. It is defined as the geometric mean of the horizontally traveled distance $d$ and the jumping height $h=h_{\max }-\bar{h}$ of the considered stride, where $\bar{h}$ denotes the height of the main body in static stance, i.e., $d_{\text {jump }}=\sqrt{d^{2}+h^{2}}$. To this end, the controller parameters are tuned manually to maximize the jumping distance $d_{\text {jump }}$ : the landing angle is selected to be $\alpha_{1}=-0.04 \mathrm{rad}$, the initial rest length is set to $r_{0}=0.15 \mathrm{~m}$, the modal switching amplitude and threshold are chosen to be $\hat{s}=0.6 \mathrm{rad}$ and $\epsilon_{\tau_{\mathrm{w}}}=0.35 \mathrm{Nm}$, respectively. This results in a jumping distance of $d_{\text {jump }}=0.107 \mathrm{~m}$, as indicated in Fig. 7(a).

\section{B. Trotting}

The experiment presented in the following aims at validating the performance of the natural dynamics based trot control. Thereby the focus is on steady state locomotion. In order to approach a phase with approximately constant movement velocity, the polar angle of the swing leg pair is initially increased step-by-step until a desired value is reached, i. e., $\alpha_{\text {sw }}=0.025 \mathrm{rad}+j 0.005 \mathrm{rad} \leq 0.525 \mathrm{rad}$, where $j \in \mathbb{N}$ iterates over the steps. All remaining controller parameters are selected as a function of $\alpha_{\mathrm{sw}}$ : To exploit the natural radial oscillation properties, the polar equilibrium angle of the stance legs is set to $\alpha_{\mathrm{st}}=1.25 \alpha_{\mathrm{sw}}$. The displacement of the initial rest length is selected by $\hat{r}=0.035 \mathrm{~m}+0.03 \frac{\mathrm{m}}{\mathrm{rad}} \alpha_{\mathrm{sw}}$. Especially, it turned out that a larger displacement for the hind leg, i.e., $\hat{r}_{\text {hind }}=1.3 \hat{r}$ while $\hat{r}_{\text {fore }}=\hat{r}$, has a stabilizing effect on the pitch motion of the main body. The thresholds of the swing leg extension and stance leg flexion are chosen to be $\epsilon_{\alpha_{\mathrm{ex}}}=0.1 \alpha_{\mathrm{sw}}$ and $\epsilon_{\alpha_{\mathrm{fl}}}=0.3 \alpha_{\mathrm{sw}}$, respectively, such that both switchings occur after mid-stance, while the swing legs extend before the stance legs flex. The threshold for the interchange of swing and stance leg pairs is selected as a quadratic function of the static polar angle of the legs in swing (with minimum at $\alpha_{\mathrm{sw}}=0$ ), i. e., $\epsilon_{\alpha}=0.6 \alpha_{\mathrm{sw}}+0.3 \frac{1}{\mathrm{rad}} \alpha_{\mathrm{sw}}^{2}$. This results in a locomotion velocity (as averaged over three steps) of $v_{\text {mean }}=0.66 \mathrm{~m} / \mathrm{s}$ (Fig. 7(b)). Fig. 7(b) shows also the commanded motor positions, gait events (triggering the FSM), and the resulting joint motion, both expressed in polar coordinates of the hind legs. It can be seen that stable (without falling) trotting is achieved, although the movement of the legs is asymmetric, which is a result of imprecisions in the mechanical realization.

\section{Dynamic walking}

The dynamic walking control performs for a wide range of nominal step lengths $\alpha_{\text {st }}$ and angles of attack $\alpha_{\mathrm{a}}$, while the threshold for the swing leg needs to be chosen depending on the angle of attack, e.g., $\epsilon_{\alpha}=0.25 \alpha_{\mathrm{a}}$. The nominal rest length $r_{0}$ and the decrease in rest length $\hat{r}$ depend on the weight of the robot and the stiffness of the SEA springs. In Fig. 7(c), the experimental results for parameters $\alpha_{\text {st }}=25 \frac{\pi}{180} \mathrm{rad}$, $\alpha_{\mathrm{a}}=-4 \frac{\pi}{180} \mathrm{rad}, r_{0}=0.152 \mathrm{~m}$, and $\hat{r}=0.06 \mathrm{~m}$ are shown as an example. This results in a locomotion velocity (as averaged over three steps) of $v_{\text {mean }}=0.25 \mathrm{~m} / \mathrm{s}$. Additionally, the 

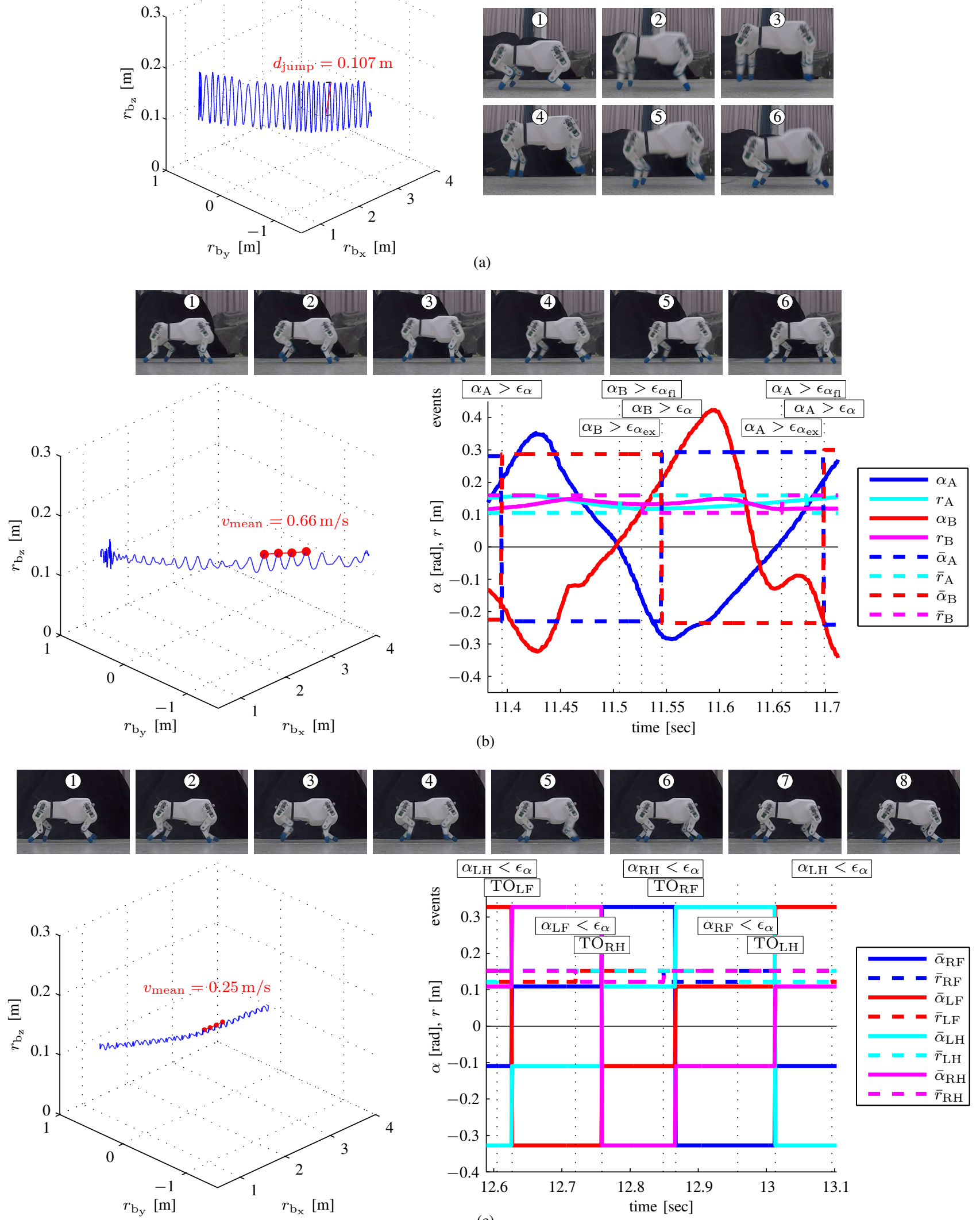

(c)

Fig. 7. Performance of locomotion controllers tested in experiments with the proposed compliantly actuated quadruped. The absolute position of the main body $\boldsymbol{r}_{\mathrm{b}}$ is plotted for (a) pronking; (b) trotting; (c) dynamic walking. The commanded motor positions (expressed in polar coordinates, i. e., leg angle $\bar{\alpha}_{i}$ and length $\bar{r}_{i}$ ) and gait events are shown for (b) trotting and (c) dynamic walking. In (b) also the measured joint motion is depicted. 
commanded motor positions expressed in polar coordinates of the legs and gait events (triggering the FSM) are shown. It can be observed that durations of symmetric gait phases such as the extension of the RF leg (finite state between events $\alpha_{\mathrm{LF}}<\epsilon_{\alpha}$ and $\mathrm{TO}_{\mathrm{RH}}$ ) and the extension of the LF leg (finite state between events $\alpha_{\mathrm{RF}}<\epsilon_{\alpha}$ and $\mathrm{TO}_{\mathrm{LH}}$ ) are not equal. This is as gait events are state-dependend and the plant is mechanically not ideally symmetric. Nevertheless the proposed approach allows to achive a stable dynamic walking gait.

\section{CONCLUSION}

In this paper, a compliantly actuated articulated leg and a quadrupedal system are introduced, which have the dynamics of the SLIP model (with polar spring) embodied in the mechanical design. This allows us to realize simple and robust quadrupedal gaits such as pronking, trotting, and dynamic walking for robotic systems with low performance (electrical) drives subject to significant imprecisions in the mechanical implementation, as validated by experiments. The embodiment of SLIP dynamics in articualted legs has been proposed in [29] for a bipedal robot. Thereby, the SLIP model properties have been validated by numerical simulation of the dynamics; the advantages regarding the generation of bipedal gaits have been verified by experiments. In this paper, our recently proposed theory of eigenmodes of nonlinear dynamics [23] is applied to mathematically show and experimentally validate that a low-dimensional fundamental model of dynamic legged locomotion can be anchored in a compliantly actuated quadruped as an example of a high-dimensional articulated multibody system with elasticities.

\section{APPENDIX}

Consider the dynamics of the form

$$
\boldsymbol{M}(\boldsymbol{q}) \ddot{\boldsymbol{q}}+\boldsymbol{b}(\boldsymbol{q}, \dot{\boldsymbol{q}})=-(\partial U(\boldsymbol{q}) / \partial \boldsymbol{q})^{T} .
$$

Herein, $\boldsymbol{q} \in \mathbb{R}^{n}$ denotes configuration variables defined in Euclidean space, $M \in \mathbb{R}^{n \times n}$ is the symmetric and p.d. inertia matrix, the generalized Coriolis/centrifugal and damping forces are summarized in $\boldsymbol{b}(\boldsymbol{q}, \dot{\boldsymbol{q}}) \in \mathbb{R}^{n}$, and $U(\boldsymbol{q}) \in \mathbb{R}_{\geq 0}$ comprises the gravitational and elastic potential.

Definition 1: Let $\boldsymbol{q}_{0}$ be the equilibrium configuration of the system (10) and let $\boldsymbol{w}$ be the components of a constant vector in Euclidean space. Let further $\Delta \boldsymbol{q}=\boldsymbol{q}-\boldsymbol{q}_{0}=\boldsymbol{w} z$ and $\dot{\boldsymbol{q}}=$ $\boldsymbol{w} \dot{z}$ be a displacement and a generalized velocity of amplitudes $z \in \mathbb{R}$ and $\dot{z} \in \mathbb{R}$ along $\boldsymbol{w}$, respectively. Then, $\boldsymbol{w}=$ const. is an eigenvector of (10), if for any $z \in \mathbb{R}$ and $\dot{z} \in \mathbb{R} \exists \ddot{z}=$ $\ddot{z}(z, \dot{z}) \in \mathbb{R}$ such that $\boldsymbol{M}\left(\boldsymbol{q}_{0}+\boldsymbol{w} z\right) \boldsymbol{w} \ddot{z}=-\boldsymbol{b}\left(\boldsymbol{q}_{0}+\boldsymbol{w} z, \boldsymbol{w} \dot{z}\right)-$ $\left.\frac{\partial U(\boldsymbol{q})}{\partial \boldsymbol{q}}\right|_{\boldsymbol{q}=\boldsymbol{q}_{0}+\boldsymbol{w} z} ^{T}$ is satisfied. An eigenvector $\boldsymbol{w}$ with $\|\boldsymbol{w}\|^{2}=1$ defines a linear transformation of the form $\Delta \boldsymbol{q}=\boldsymbol{q}-\boldsymbol{q}_{0}=\boldsymbol{w} z$ and $\dot{\boldsymbol{q}}=\boldsymbol{w} \dot{z}$. The motion of (10), which can be parameterized exclusively by $z$ and $\dot{z}$, is referred to as eigenmode of (10).

\section{REFERENCES}

[1] R. M. Alexander, "Three uses for springs in legged locomotion," Int. J. of Robotics Research, vol. 9, no. 2, pp. 53-61, 1990.

[2] R. Blickhan, "The spring-mass model for running and hopping," $J$. of Biomechanics, vol. 22, pp. 1217-1227, 1989.

[3] A. Seyfarth, H. Geyer, M. Günther, and R. Blickhan, "A movement criterion for running." J. of Biomechanics, vol. 35 , no. 5, pp. 649-55, 2002.
[4] H. Geyer, A. Seyfarth, and R. Blickhan, "Compliant leg behavior explains basic dynamics of walking and running," Proc. R. Soc. B, vol. 273, no. 1603, pp. 2861-2867, 2006.

[5] H.-M. Maus, J. Rummel, and A. Seyfarth, "Stable upright walking and running using a simple pendulum based control scheme," in Proc. Int. Conf. Climbing and Walking Robots, 2008, pp. 623-629.

[6] H.-M. Maus, S. Lipfert, M. Gross, J. Rummel, and A. Seyfarth, "Upright human gait did not provide a major mechanical challenge for our ancestors," Nature communications, vol. 1, p. 70, 2010.

[7] J. Rummel, Y. Blum, H. M. Maus, C. Rode, and A. Seyfarth, "Stable and robust walking with compliant legs," in Proc. of IEEE Int. Conf. on Robotics and Automation, 2010, pp. 5250-5255.

[8] R. Full and D. Koditschek, "Templates and anchors: neuromechanical hypotheses of legged locomotion on land," J. Exp. Biol., vol. 202, no. 15, pp. 3325-3332, 1999

[9] P. Holmes, R. Full, D. Koditschek, and J. Guckenheimer, "The dynamics of legged locomotion: Models, analyses and challenges," SIAM Review, vol. 48, no. 2, pp. 207-304, 2006.

[10] M. H. Raibert, "Four-legged running with one-legged algorithms," in Int. Symp. on Robotics Research. Cambridge: MIT Press, 1985, pp. 311-315.

[11] M. Raibert, K. Blankespoor, G. Nelson, R. Playter, and B. Team, "Bigdog, the rough-terrain quadruped robot," in Proc. of the World Congress, vol. 17, no. 1, 2008, pp. 10822-10825.

[12] C. Semini, N. G. Tsagarakis, E. Guglielmino, M. Focchi, F. Cannella, and D. G. Caldwell, "Design of hyq-a hydraulically and electrically actuated quadruped robot," Proc. of the Inst. of Mech. Eng., Part I: J. of Systems and Control Engineering, vol. 225, pp. 831-849, 2011.

[13] C. Semini, V. Barasuol, T. Boaventura, M. Frigerio, M. Focchi, D. G. Caldwell, and J. Buchli, "Towards versatile legged robots through active impedance control," Int. J. of Robotics Research, vol. 34, no. 7, pp. $1003-1020,2015$

[14] S. Seok, A. Wang, M. Y. M. Chuah, D. J. Hyun, J. Lee, D. M. Otten, J. H. Lang, and S. Kim, "Design principles for energy-efficient legged locomotion and implementation on the mit cheetah robot," Trans. on Mechatronics, vol. 20, no. 3, pp. 1117-1129, 2015.

[15] A. Sproewitz, A. Tuleu, M. Vespignani, M. Ajallooeian, E. Badri, and A. Ijspeert, "Towards Dynamic Trot Gait Locomotion-Design, Control and Experiments with Cheetah-cub, a Compliant Quadruped Robot," Int. J. of Robotics Research, vol. 32, no. 8, pp. 932-950, 2013

[16] M. Hutter, C. Gehring, M. Bloesch, M. Hoepflinger, C. D. Remy, and R. Siegwart, "Starleth: A compliant quadrupedal robot for fast, efficient, and versatile locomotion," in Int. Conf. on Climbing and Walking Robots, 2012, pp. 483-490.

[17] M. Hutter, C. Gehring, D. Jud, A. Lauber, C. D. Bellicoso, V. Tsounis, J. Hwangbo, K. Bodie, P. Fankhauser, M. Bloesch, et al., "Anymal-a highly mobile and dynamic quadrupedal robot," in IEEE/RSJ Int. Conf. on Intelligent Robots and Systems. IEEE, 2016, pp. 38-44.

[18] M. H. Raibert, Legged Robots That Balance. MIT Press, 1986.

[19] — " "Trotting, pacing and bounding by a quadruped robot," J. of Biomechanics, vol. 23, pp. 7983-8198, 1990.

[20] C. Gehring, S. Coros, M. Hutter, M. Bloesch, M. Hoepflinger, and R. Siegwart, "Control of dynamic gaits for a quadrupedal robot," in IEEE Int. Conf. on Robotics and Automation, May 2013, pp. 32873292.

[21] M. Hutter, C. Gehring, M. A. Höpflinger, M. Blösch, and R. Siegwart, "Toward combining speed, efficiency, versatility, and robustness in an autonomous quadruped." IEEE Trans. Robotics, vol. 30, no. 6, pp. 1427 $1440,2014$.

[22] C. Gehring, S. Coros, M. Hutter, M. Bloesch, P. Fankhauser, M. A. Hoepflinger, and R. Siegwart, "Towards automatic discovery of agile gaits for quadrupedal robots," in IEEE Int. Conf. on Robotics and Automation. IEEE, 2014, pp. 4243-4248.

[23] D. Lakatos, W. Friedl, and A. Albu-Schaffer, "Eigenmodes of nonlinear dynamics: Definition, existence, and embodiment into legged robots with elastic elements," IEEE Robotics and Automation Letters, vol. 2, no. 2, pp. 1062-1069, Apr. 2017

[24] J. Grizzle, J. Hurst, B. Morris, H.-W. Park, and K. Sreenath, "MABEL a new robotic bipedal walker and runner," in American Control Conf. IEEE, 2009, pp. 2030-2036.

[25] R. Blickhan and R. Full, "Similarity in multilegged locomotion: bouncing like a monopode," J. of Comparative Physiology A, vol. 173, no. 5, pp. $509-517,1993$

[26] D. Lakatos, D. Seidel, W. Friedl, and A. Albu-Schäffer, "Targeted jumping of compliantly actuated hoppers based on discrete planning and switching control," in Proc. IEEE/RSJ Int. Conf. on Intelligent Robots and Systems, 2015, pp. 5802-5808.

[27] D. F. Hoyt and C. R. Taylor, "Gait and the energetics of locomotion in horses." Nature, 1981.

[28] J. Pratt, P. Dilworth, and G. Pratt, "Virtual model control of a bipedal walking robot," in IEEE Int. Conf. on Robotics and Automation, 1997, pp. $193-198$.

[29] C. Hubicki, J. Grimes, M. Jones, D. Renjewski, A. Sproewitz, A. Abate, and J. Hurst, "Atrias: Design and validation of a tether-free 3d-capable spring-mass bipedal robot," Int. J. of Robotics Research, vol. 35, no. 12 pp. 1497-1521, 2016. 